\title{
Maximizing the benefits of ART and PrEP in resource- limited settings
}

\author{
G. AKUDIBILLAH ${ }^{1 *}$, A. PANDEY ${ }^{2}$ AND J. MEDLOCK ${ }^{3}$ \\ ${ }^{1}$ Department of Environmental Sciences, Oregon State University, Corvallis, OR, USA \\ ${ }^{2}$ Center for Infectious Disease Modeling and Analysis, Yale University, New Haven, CT, USA \\ ${ }^{3}$ Department of Biomedical Sciences, Oregon State University, Corvallis, OR, USA
}

Received 3 October 2016; Accepted 9 November 2016;

first published online 29 December 2016

\section{SUMMARY}

Antiretroviral therapy (ART) is increasingly being used as an HIV-prevention tool, administered to uninfected people with ongoing HIV exposure as pre-exposure prophylaxis (PrEP) and to infected people to reduce their infectiousness. We used a modelling approach to determine the optimal population-level combination of ART and PrEP allocations required in South Africa to maximize programme effectiveness for four outcome measures: new infections, infection-years, death and cost. We considered two different strategies for allocating treatment, one that selectively allocates drugs to sex workers and one that does not. We found that for low treatment availability, prevention through PrEP to the general population or PrEP and ART to sex workers is key to maximizing effectiveness, while for higher drug availability, ART to the general population is optimal. At South Africa's current level of treatment availability, using prevention is most effective at reducing new infections, infection-years, and cost, while using the treatment as ART to the general population best reduces deaths. At treatment levels that meet the UNAIDS's ambitious new 90-90-90 target, using all or almost all treatment as ART to the general population best reduces all four outcome measures considered.

Key words: ART, HIV/AIDS, mathematical modelling, optimization, PrEP.

\section{INTRODUCTION}

The Joint United Nations Programme on HIV and AIDS (UNAIDS) estimates that 31.6-35.2 million people are living with HIV worldwide, with 2.4-2.9 million new HIV infections and 1.6-1.9 million AIDS-related deaths in 2010 [1]. HIV/AIDS is a major problem in developing countries especially in Sub-Saharan Africa. This region constitutes $12 \%$ of the world's population, yet accounts for $72 \%$ of AIDS-related deaths [1]. In

\footnotetext{
* Author for correspondence: Dr G. Akudibillah, 106 Dryden Hall, Corvallis, Oregon 97331, USA.

(Email: akudibig@oregonstate.edu)
}

Sub-Saharan Africa, most HIV transmission in adults occurs from heterosexual sex, with commercial sex [2] and multiple partners being key drivers of HIV transmission [3, 4].

Antiretroviral therapy (ART) has been traditionally used to prevent progression from HIV to AIDS [5, 6]. Recently, antiretroviral drugs have been shown to have the additional benefit of substantially reducing the infectiousness of infected people, leading to reduced transmission [7-9]. The HPTN052 trials showed that early treatment is able to reduce heterosexual transmission of HIV in serodiscordant couples by as much as $96 \%$ [10]. 
ART is also administered to uninfected people either as post-exposure prophylaxis (PEP) after highrisk exposure or as pre-exposure prophylaxis (PrEP) for people with ongoing HIV exposure [11-13]. Giving ART as PrEP to uninfected heterosexual people reduces the chance of acquiring HIV by $63-$ $73 \%$ [12]. The US Centers for Disease Control and Prevention (CDC) recommends the use of ART as PrEP to, among others, serodiscordant couples and homosexual men or women who do not always use condoms when having sex with partners known to be at risk for HIV [14].

Whether as treatment to reduce the infectivity of infected people or as prophylaxis to reduce the susceptibility of uninfected people, ART has thus become an important strategy in the fight against HIV transmission [15-18]. To take advantage of the benefits of antiretroviral theraphy, the World Health Organization (WHO) revised its treatment guidelines in 2013, recommending that treatment be initiated in infected adults and adolescents with a CD4 count of $\leqslant 500$ cells $/ \mathrm{mm}^{3}[19]$. UNAIDS also recently introduced an ambitious treatment target called 90-90-90, which by 2020 aims to have $90 \%$ of all people living with HIV know their HIV status, $90 \%$ of all people with diagnosed HIV infection receive sustained ART and $90 \%$ of all people receiving ART have viral suppression [20].

In this paper, we aim to inform drug-allocation policy in resource-limited settings by using a compartmental mathematical model for heterosexual transmission of HIV with treatment targeted by infection status, sexual-activity level and gender. Due to the availability of high-quality data, the model was parameterized for South Africa. Under South Africa's current policy, treatment is not stratified by gender or risk, and no PrEP is given.

We used optimization methods to determine the allocations of ART in the target groups that minimize new infections, HIV-related deaths and cost. We examined treatment allocation given fixed amounts of treatment (or, equivalently, fixed cost of treatment) to understand the trade-offs from giving ART and PrEP to different groups. We also examined the effect of increasing the amount of drugs available on new infections and prevalence. Moreover, we compared our optimization results to the $90-90-90$ policy. We also evaluated the sensitivity of our results to parameter uncertainty across a range of infection costs, new infection deaths and cost.

\section{METHODS}

\section{Model}

We constructed a mathematical model that incorporates risk, gender and treatment (Fig. 1). We briefly outline the model here and the Supplementary material contains a detailed description of the model.

Our model stratifies the population by gender, level of sexual activity, HIV status and treatment status (Fig. 1). The female population is divided into low, medium and high risk, while the male population is divided into low and medium risk. Low-risk people are in monogamous marriages, single or otherwise have zero or one sexual partner. Medium-risk people have more than one sexual partner. High-risk females are commercial sex workers, sometimes called transactional sex workers. We neglected the high-risk class for males because male and transgender commercial sex workers in Sub-Saharan Africa are rare [21]. Each of the risk groups is further stratified by HIV infection status (i.e. HIV negative and HIV positive) and by treatment status (treated and untreated).

The model only considers the adult population. People enter the model at per capita rate $b$, when they attain the age of 15 years, as low risk and leave either through natural death, with per capita rate $\mu$, or death due to AIDS, with per capita rate $\gamma$. Movement between risk groups is due to changes in sexual behaviour and movement within risk groups is due to either infection or people electing to start or stop treatment. HIV-negative people can only become infected by heterosexual sexual contact; men who have sex with men, injecting drug use and vertical transmission are not included in the model.

We assumed perfect adherence to treatment, i.e. individuals who start using treatment will strictly adhere the prescribed regimen until they decide to stop treatment. All other HIV-prevention measures such as male circumcision, education and condom use are aggregated in the HIV risk per sex act and are assumed constant for the span of the model. While HIV-induced death rate depends on disease stage, typically measured by CD4 count or viral load $[22,23]$, we however used a death rate $\gamma$ that is averaged over disease stage to facilitate understanding of the model results and to reduce the computational burden of generating the results. 

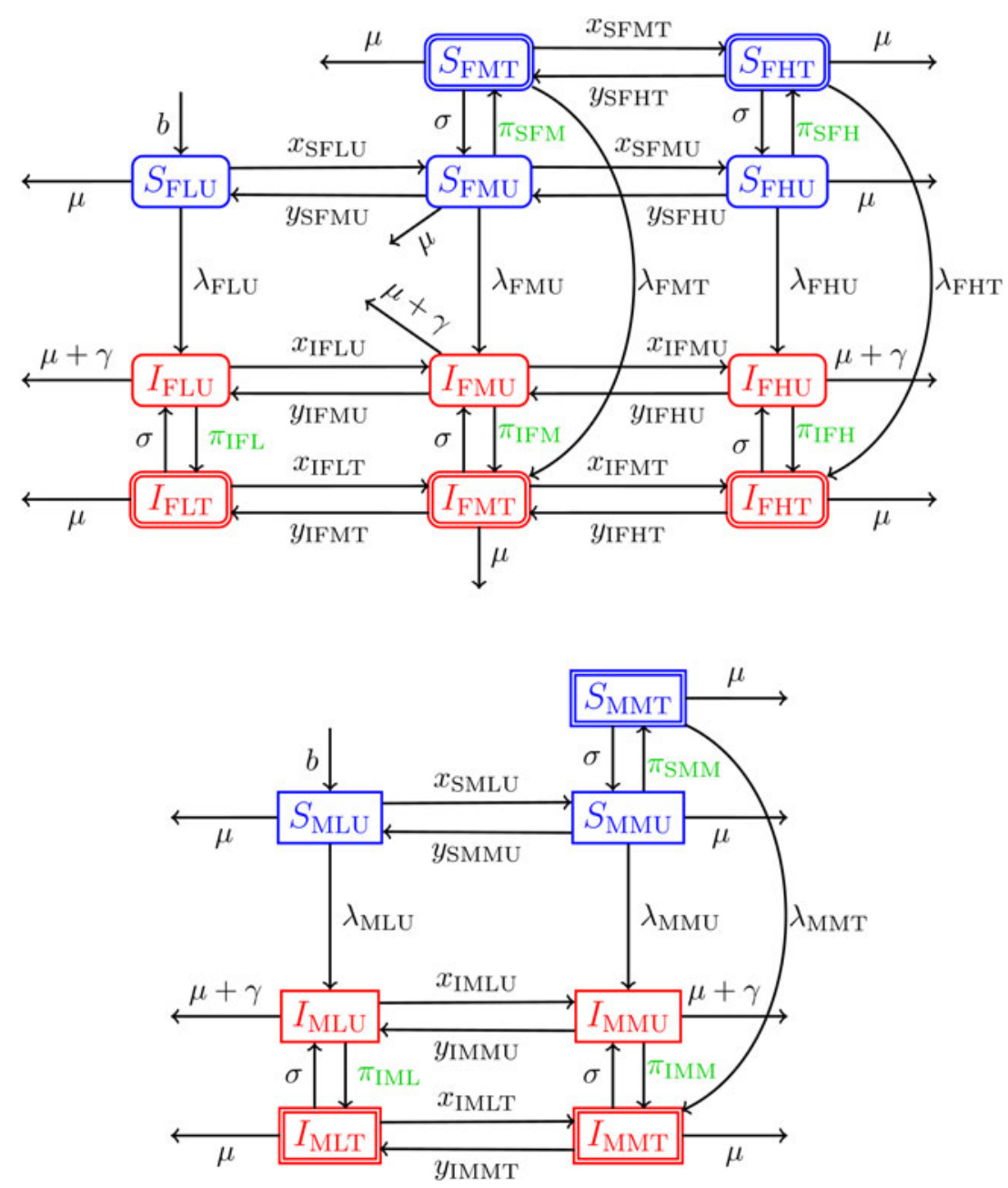

Fig. 1. Diagram of HIV model. HIV-infected people are in red font and susceptible (i.e. uninfected) people are in blue font. The first subscript on the state variables $S$ and $I$ denotes gender (female or male), the second denotes risk level (low, medium, high), and the last denotes treatment status (untreated or treated). The subscripts on the other variables and parameters have similar meanings. The variables effected by treatment interventions are in green font.

The forces of infection for females and males are

$$
\left.\begin{array}{l}
\lambda_{\mathrm{F} i j}(t)=\sum_{k \in\{\mathrm{L}, \mathrm{M}\} \ell \in\{\mathrm{U}, \mathrm{T}\}} \psi c_{i j k \ell} \frac{I_{\mathrm{M} k \ell}(t)}{N(t)}, \quad \text { for } i \in\{\mathrm{L}, \mathrm{M}, \mathrm{H}\} \text { and } j \in\{\mathrm{U}, \mathrm{T}\}, \\
\lambda_{\mathrm{M} i j}(t)=\sum_{k \in\{\mathrm{L}, \mathrm{M}, \mathrm{H}\} \ell \in\{\mathrm{U}, \mathrm{T}\}} c_{i j k \ell} \frac{I_{\mathrm{F} k l}(t)}{N(t)}, \quad \text { for } i \in\{\mathrm{L}, \mathrm{M}\} \text { and } j \in\{\mathrm{U}, \mathrm{T}\},
\end{array}\right\}
$$

where the total population size is

$$
N(t)=\sum_{\substack{i \in\{\mathrm{F}, \mathrm{M}\} \\ j \in\{\mathrm{L}, \mathrm{M}, \mathrm{H}\} \\ k \in\{\mathrm{U}, \mathrm{T}\}}}\left[S_{i j k}(t)+I_{i j k}(t)\right],
$$

with $S_{i j k}$ and $I_{i j k}$ defined to be 0 for the groups not in the model (FLT, MLT, MHU, and MHT), and the transmission rate is

$c_{i j k \ell}=d_{i k} \cdot e_{j} \cdot f_{\ell}$, 
with the infectivity set by the riskiest partner,

$d_{i k}= \begin{cases}\beta_{\mathrm{L}} & \text { if } i=k=\mathrm{L}, \\ \beta_{\mathrm{M}} & \text { if } i=\mathrm{M} \text { or }(k=\mathrm{M} \text { and } i \neq \mathrm{H}), \\ \beta_{\mathrm{H}} & \text { if } i=\mathrm{H},\end{cases}$

the transmissibility reduced if the uninfected partner is on PrEP,

$e_{j}= \begin{cases}1 & \text { if } j=\mathrm{U}, \\ 1-\theta_{\operatorname{PrEP}} & \text { if } j=\mathrm{T},\end{cases}$

and the transmissibility is reduced if the infected partner is on ART

$f_{\ell}= \begin{cases}1 & \text { if } \ell=\mathrm{U}, \\ 1-\theta_{\mathrm{ART}} & \text { if } \ell=\mathrm{T} .\end{cases}$

The model is specified as the system of differential equations

$$
\begin{aligned}
\frac{\mathrm{d} S_{\mathrm{FLU}}}{\mathrm{d} t}= & \frac{b N}{2}+y_{\mathrm{SFMU}} F_{\mathrm{NMU}}-\left(x_{\mathrm{SFLU}}+\lambda_{\mathrm{FLU}}\right. \\
& +\mu) S_{\mathrm{FLU}},
\end{aligned}
$$

$\frac{\mathrm{d} I_{\mathrm{FLU}}}{\mathrm{d} t}=\lambda_{\mathrm{FLU}} S_{\mathrm{FLU}}+y_{\mathrm{IFMU}} I_{\mathrm{FMU}}+\sigma I_{\mathrm{FLT}}$ $-\left(x_{\mathrm{IFLU}}+\pi_{\mathrm{IML}}+\gamma+\mu\right) I_{\mathrm{FLU}}$,

$$
\begin{aligned}
\frac{\mathrm{d} I_{\mathrm{FLT}}}{\mathrm{d} t}= & y_{\mathrm{IFMT}} I_{\mathrm{FMT}}+\pi_{\mathrm{IFL}} F_{\mathrm{PLU}}-\left(x_{\mathrm{IFLT}}+\sigma\right. \\
& +\mu) I_{\mathrm{FLT}},
\end{aligned}
$$

$$
\begin{aligned}
\frac{\mathrm{d} S_{\mathrm{MLU}}}{\mathrm{d} t}= & \frac{b N}{2}+y_{\mathrm{SMMU}} S_{\mathrm{MMU}}-\left(x_{\mathrm{SMLU}}+\lambda_{\mathrm{MLU}}\right. \\
& +\mu) S_{\mathrm{MLU}},
\end{aligned}
$$

$$
\begin{aligned}
\frac{\mathrm{d} I_{\mathrm{MLU}}}{\mathrm{d} t}= & y_{\mathrm{IMMU}} I_{\mathrm{MMU}}+\lambda_{\mathrm{MLU}} S_{\mathrm{MLU}}+\sigma I_{\mathrm{MLT}} \\
& -\left(x_{\mathrm{IMLU}}+\pi_{\mathrm{IML}}+\gamma+\mu\right) I_{\mathrm{MLU}},
\end{aligned}
$$$$
\frac{\mathrm{d} I_{\mathrm{MLT}}}{\mathrm{d} t}=y_{I M M T} I_{\mathrm{MMT}}+\pi_{\mathrm{IML}} I_{\mathrm{MLU}}-\left(x_{\mathrm{IMLT}}\right.
$$$$
+\sigma+\mu) I_{\mathrm{MLT}},
$$

$$
\begin{aligned}
\frac{\mathrm{d} S_{\mathrm{FMU}}}{\mathrm{d} t}= & x_{\mathrm{SFLU}} S_{\mathrm{FLU}}+y_{\mathrm{SFHU}} S_{\mathrm{FHU}}+\sigma S_{\mathrm{FMT}} \\
& -\left(y_{\mathrm{SFMU}}+x_{\mathrm{SFMU}}+\pi_{\mathrm{SFM}}+\lambda_{\mathrm{FMU}}\right. \\
& +\mu) S_{\mathrm{FMU}},
\end{aligned}
$$

$$
\begin{aligned}
\frac{\mathrm{d} S_{\mathrm{FMT}}}{\mathrm{d} t}= & y_{\mathrm{SFHT}} S_{\mathrm{FHT}}+\pi_{\mathrm{SFM}} S_{\mathrm{FMU}}-\left(x_{\mathrm{SFMT}}\right. \\
& \left.+\sigma+\lambda_{\mathrm{FMT}}+\mu\right) S_{\mathrm{FMT}}
\end{aligned}
$$

$$
\begin{aligned}
\frac{\mathrm{d} I_{\mathrm{FHT}}}{\mathrm{d} t}= & x_{\mathrm{IFMT}} I_{\mathrm{FMT}}+\pi_{\mathrm{IFH}} I_{\mathrm{FHU}}+\lambda_{\mathrm{FHT}} S_{\mathrm{FHT}} \\
& -\left(y_{\mathrm{IFHT}}+\sigma+\mu\right) I_{\mathrm{FHT}} .
\end{aligned}
$$

\section{Parameterization}

We parameterized our model with demographic data from South Africa because of the availability of excellent demographic statistics (Tables 1-3). Our model is initialized for the beginning of 2014 $(t=0)$, although we used demographic data from 2012, the date of the last South African National HIV Prevalence, Incidence and Behavior Survey [24]. 
Table 1. Model parameters (see also Tables 2 and 3)

\begin{tabular}{llll}
\hline \hline Parameter & Description & Value & Source \\
\hline$b$ & Birth rate & $0 \cdot 0226 \mathrm{yr}^{-1}$ & {$[25]$} \\
$\mu$ & Natural death rate & $0 \cdot 0106 \mathrm{yr}^{-1}$ & {$[25]$} \\
$\gamma$ & Death rate due to AIDS & $0 \cdot 0909 \mathrm{yr}^{-1}$ & {$[1]$} \\
$\beta_{\mathrm{L}}$ & Low-risk contact rate & $0 \cdot 0050 \mathrm{yr}^{-1}$ & {$[9]$} \\
$\beta_{\mathrm{M}}$ & Medium-risk contact rate & $0 \cdot 0075 \mathrm{yr}^{-1}$ & - \\
$\beta_{\mathrm{H}}$ & High-risk contact rate & $0 \cdot 0250 \mathrm{yr}^{-1}$ & {$[26]$} \\
$\psi$ & Male-to-female relative transmission rate & 2 & {$[27]$} \\
$\theta_{\mathrm{ART}}$ & Efficacy of ART at preventing transmission & $96 \%$ & {$[12]$} \\
$\theta_{\mathrm{PrEP}}$ & Efficacy of PrEP at preventing transmission & $71 \%$ & - \\
$r_{\mathrm{max}}$ & Rate of enrolling people on treatment & $20 \mathrm{yr}^{-1} \mathrm{treatment}^{-1}$ & {$[28]$} \\
$c_{\mathrm{I}}$ & Cost of an infection & $\$ 1000 \mathrm{yr}^{-1}$ & - \\
$c_{\mathrm{D}}$ & Cost of a death & $\$ 100000$ & {$[29]$} \\
$c_{\mathrm{T}}$ & Cost of treatment & $\$ 120 \mathrm{yr}^{-1}$ & {$[30]$} \\
$r$ & Discount rate for costs & $3 \% \mathrm{yr}^{-1}$ & {$[24]$} \\
$\sigma$ & Rate of treatment stoppage & $0 \cdot 2 \mathrm{yr}^{-1}$ & {$[24]$} \\
$\phi_{\mathrm{FL}}$ & Initial prevalence in low-risk females & $14 \cdot 4 \%$ & {$[24]$} \\
$\phi_{\mathrm{FM}}$ & Initial prevalence in medium-risk females & $23 \cdot 2 \%$ & {$[24]$} \\
$\phi_{\mathrm{FH}}$ & Initial prevalence in high-risk females & $59 \cdot 6 \%$ & {$[24]$} \\
$\phi_{\mathrm{ML}}$ & Initial prevalence in low-risk males & $17 \cdot 3 \%$ & {$[24]$} \\
$\phi_{\mathrm{MM}}$ & Initial prevalence in medium-risk males & $14 \cdot 5 \%$ & \\
\hline \hline
\end{tabular}

Table 2. Model initial conditions

\begin{tabular}{ll}
\hline \hline Variable & Value \\
\hline$S_{\text {FLU }}(0)$ & 14767171 \\
$I_{\text {FLU }}(0)$ & 2017210 \\
$I_{\mathrm{FLT}}(0)$ & 1071932 \\
$S_{\mathrm{MLU}}(0)$ & 11865435 \\
$I_{\mathrm{MLU}}(0)$ & 1764793 \\
$I_{\mathrm{MLT}}(0)$ & 717335 \\
$S_{\mathrm{FMU}}(0)$ & 719587 \\
$S_{\mathrm{FMT}}(0)$ & 0 \\
$I_{\mathrm{FMU}}(0)$ & 98296 \\
$I_{\mathrm{FMT}}(0)$ & 52234 \\
$S_{\mathrm{MMU}}(0)$ & 2984922 \\
$S_{\mathrm{MMT}}(0)$ & 0 \\
$I_{\mathrm{MMU}}(0)$ & 443959 \\
$I_{\mathrm{MMT}}(0)$ & 180456 \\
$S_{\mathrm{FHU}}(0)$ & 76419 \\
$S_{\mathrm{FHT}}(0)$ & 0 \\
$I_{\mathrm{FHU}}(0)$ & 73617 \\
$I_{\mathrm{FHT}}(0)$ & 39120 \\
\hline \hline
\end{tabular}

The initial numbers of people in each compartment (Table 2) were derived from separate South African data on the current prevalence by gender and risk and on the proportion of infected people currently receiving ART. From the 2012 South African National HIV Prevalence, Incidence and Behavior Survey [24], adults who reported having one sexual partner in the last 12 months were considered low risk. Multiple sexual partners increases the likelihood of exposure to HIV through expanding sexual networks, so people who reported having more than one sexual partner in the last 12 months were classified as medium risk. Female sex workers were considered as the high-risk population. The number of people in each gender and risk group, combined with the current prevalence by gender and risk from the same survey, were used to determine the initial number of people infected and susceptible:

$I_{i j}(0)=\phi_{i j} N_{i j}(0), \quad S_{i j}(0)=\left(1-\phi_{i j}\right) N_{i j}(0)$,

for $i \in\{\mathrm{F}, \mathrm{M}\}$ and $j \in\{\mathrm{L}, \mathrm{M}, \mathrm{H}\}$, where $\phi_{i j}$ is the prevalence and $N_{i j}(0)$ is the initial number of people, both susceptible and infected, in that gender and risk group. We then initialized the number of infected people on ART in each gender and risk group to $42 \%$, the current proportion of infected people on ART in South Africa [1]:

$I_{i j \mathrm{~T}}(0)=0.42 I_{i j}(0), \quad I_{i j \mathrm{U}}(0)=(1-0.42) I_{i j}(0)$,

for $i \in\{\mathrm{F}, \mathrm{M}\}$ and $j \in\{\mathrm{L}, \mathrm{M}, \mathrm{H}\}$. We further assumed no widespread use of PrEP, i.e. $S_{i j \mathrm{~T}}(0)=0$ for $i \in\{\mathrm{F}, \mathrm{M}\}$ and $j \in\{\mathrm{L}, \mathrm{M}, \mathrm{H}\}$.

We took the low-risk contact rate, $\beta_{\mathrm{L}}$, to be the annual risk for serodiscordant couples [9], and then the medium- and high-risk contact rates, $\beta_{\mathrm{M}}$ and $\beta_{\mathrm{H}}$, 
Table 3. Model risk transition rates

\begin{tabular}{lll}
\hline \hline Parameter & Risk transition rate & Value \\
\hline$x_{\text {SFLU }}$ & From low to medium risk for untreated susceptible females & $0 \cdot 05 \mathrm{yr}^{-1}$ \\
$x_{\text {SFMU }}$ & From medium to high risk for untreated susceptible females & $0 \cdot 06 \mathrm{yr}^{-1}$ \\
$y_{\text {SFMU }}$ & From medium to low risk for untreated susceptible females & $0 \cdot 02 \mathrm{yr}^{-1}$ \\
$x_{\text {SFMT }}$ & From medium to high risk for treated susceptible females & $0 \cdot 05 \mathrm{yr}^{-1}$ \\
$y_{\text {SFHU }}$ & From high to medium risk for untreated susceptible females & $0 \cdot 02 \mathrm{yr}^{-1}$ \\
$y_{\text {SFHT }}$ & From high to medium risk for treated susceptible females & $0 \cdot 02 \mathrm{yr}^{-1}$ \\
$x_{\text {SMLU }}$ & From low to medium risk for untreated susceptible males & $0 \cdot 05 \mathrm{yr}^{-1}$ \\
$y_{\text {SMMU }}$ & From medium to low risk for untreated susceptible males & $0 \cdot 03 \mathrm{yr}^{-1}$ \\
$x_{\text {IFLU }}$ & From low to medium risk for untreated infected females & $0 \cdot 02 \mathrm{yr}^{-1}$ \\
$x_{\text {IFLT }}$ & From low to medium risk for treated infected females & $0 \cdot 06 \mathrm{yr}^{-1}$ \\
$x_{\text {IFMU }}$ & From medium to high risk for untreated infected females & $0 \cdot 06 \mathrm{yr}^{-1}$ \\
$y_{\text {IFMU }}$ & From medium to low risk for untreated infected females & $0 \cdot 04 \mathrm{yr}^{-1}$ \\
$x_{\text {IFMT }}$ & From medium to high risk for treated infected females & $0 \cdot 07 \mathrm{yr}^{-1}$ \\
$y_{\text {IFMT }}$ & From medium to low risk for treated infected females & $0 \cdot 02 \mathrm{yr}^{-1}$ \\
$y_{\text {IFHU }}$ & From high to medium risk for untreated infected females & $0 \cdot 07 \mathrm{yr}^{-1}$ \\
$y_{\text {IFHT }}$ & From high to medium risk for treated infected females & $0 \cdot 01 \mathrm{yr}^{-1}$ \\
$x_{\text {IMLU }}$ & From low to medium risk for untreated infected males \\
$x_{\text {IMLT }}$ & From low to medium risk for treated infected males & $0 \cdot 06 \mathrm{yr}^{-1}$ \\
$y_{\text {IMMU }}$ & From medium to low risk for untreated infected males & $0 \cdot 07 \mathrm{yr}^{-1}$ \\
$y_{\text {IMMT }}$ & From medium to low risk for treated infected males & $0 \cdot 03 \mathrm{yr}^{-1}$ \\
\hline \hline
\end{tabular}

to be respectively $50 \%$ and $400 \%$ greater than the lowrisk contact rate.

Treatment is generally constrained by number of drugs available, the manpower available to distribute the drugs and other resources. The rate of enrolling people on treatment per drug available per untreated person $\left(r_{\max }\right)$ was assumed to be $20 \mathrm{yr}^{-1}$ treatment ${ }^{-1}$, i.e. it takes about $1 / 20 \mathrm{yr}$ or about 18 days for an eligible person to get on treatment.

The cost of death $\left(c_{\mathrm{D}}\right)$ is the financial benefit that an otherwise healthy person would contribute to society on average over his or her remaining lifetime was assumed to be US\$100 000 .

Due to the absence of behavioural data, values for the rates of transition between risk groups ( $x_{i j k \ell}$ and $y_{i j k \ell}$ ) were assumptions (Table 3).

\section{Outcome measures}

We determined the proportion of each group to be treated according to four different outcome measures: the number of new infections, the number of total infection-years, the number of deaths and total cost. These were all evaluated over a time horizon of $t_{\text {end }}$ $=10 \mathrm{yr}$.

New infections. The total number of new infections is the sum of the rates of new infections arising in each of the eight uninfected classes integrated over the time period:

$$
\int_{0}^{t_{\text {end }}} \sum_{\substack{i \in\{\mathrm{F}, \mathrm{M}\} \\ j \in\{\mathrm{L}, \mathrm{M}, \mathrm{H}\} \\ k \in\{\mathrm{U}, \mathrm{T}\}}} \lambda_{i j k}(t) S_{i j k}(t) \mathrm{d} t,
$$

with $\lambda_{i j k}$ defined to be 0 for the groups not in the model (FLT, MLT, MHU, MHT).

Total infection-years. The total infection-years is the sum of the number of all the infected people (both treated and untreated) in the 10 infected classes at each time, integrated over the time period, i.e.

$$
\int_{0}^{t_{\mathrm{end}}} I(t) \mathrm{d} t
$$

where the number of infected people is

$$
I(t)=\sum_{\substack{i \in\{\mathrm{F}, \mathrm{M}\} \\ j \in\{\mathrm{L}, \mathrm{M}, \mathrm{H}\} \\ k \in\{\mathrm{U}, \mathrm{T}\}}} I_{i j k}(t) .
$$

Deaths due to AIDS. Treatment prevents progression from HIV to AIDS, thus reducing deaths. We assumed that AIDS-related deaths only occur in infected, untreated people. Thus, the total number of deaths due to AIDS is the number of untreated 
infected people at each time,

$I_{\mathrm{U}}(t)=\sum_{\substack{i \in\{\mathrm{F}, \mathrm{M}\} \\ j \in\{\mathrm{L}, \mathrm{M}, \mathrm{H}\}}} I_{i j \mathrm{U}}(t)$,

multiplied by the rate of death due to AIDS:

$\int_{0}^{t_{\text {end }}} \gamma I_{\mathrm{U}}(t) \mathrm{d} t$

Total cost. The total cost consists of cost of infections, cost of deaths, and cost of treatment. The costs of infections and HIV-related deaths depend on disease stage [31] for ease of analysis, we however used a cost of infections $C_{\mathrm{I}}(t)$ and cost of deaths $C_{\mathrm{D}}(t)$ that are averaged over disease stage. The disease cost per year, which includes monetary equivalent loss of the infected people, like lost productivity, etc., is the average cost of disease per person per year multiplied by the total number of people infected,

$C_{\mathrm{I}}(t)=c_{\mathrm{I}} I(t)$.

The cost per year of deaths is the the cost per death multiplied by the number of deaths per year,

$C_{\mathrm{D}}(t)=c_{\mathrm{D}} \gamma I_{\mathrm{U}}(t)$.

The treatment cost per year is the cost per person per year multiplied by the number of people treated:

$C_{\mathrm{T}}(t)=c_{\mathrm{T}} T(t)$,

where the number of people treated is

$$
T(t)=\sum_{\substack{i \in\{\mathrm{F}, \mathrm{M}\} \\ j \in\{\mathrm{L}, \mathrm{M}, \mathrm{H}\}}}\left[S_{i j \mathrm{~T}}(t)+I_{i j \mathrm{~T}}(t)\right] .
$$

The cost outcome measure is the discounted sum of these costs, integrated over the time period:

$\int_{0}^{t_{\text {end }}}\left[C_{\mathrm{I}}(t)+C_{\mathrm{D}}(t)+C_{\mathrm{T}}(t)\right] \mathrm{e}^{-r t} \mathrm{~d} t$

The total cost is discounted at rate $r$, representing the rate a policymaker is willing to pay as trade-off for the cost today vs. future cost [32].

\section{Controls and constraints}

We considered two different ways to model governmentlevel intervention programmes that use antiretroviral drugs to control HIV. In both intervention models, the antiretroviral drugs can either be used as treatment of infected people (ART) or as prophylaxis for uninfected people (PrEP). In the first intervention model, global ART \& PrEP, infected people are given ART and uninfected people are given PrEP at coverage levels that are independent of their risk of transmission. In the second intervention model, ART \& highrisk PrEP, there are coverage levels of ART and PrEP for high-risk people and another coverage level of ART for low- and medium-risk people, with no PrEP for low- and medium-risk people. The latter intervention model was chosen to evaluate the CDC's current recommendation that ART be given to all infected people and PrEP only be given to highrisk people [33].

The control variables are the total number of people targeted to be on treatment in each of the designated groups $\left(V_{G}\right)$ at any one time. From these treatment targets, the flows of people into treatment per unit time were taken to be

$\pi_{G}(t)=r_{\max } \max \left(V_{G}-T_{G}(t), 0\right)$,

where $T_{G}(t)$ is the total number of people in group $G$ currently on treatment. The quantity $\max \left(V_{G}-T_{G}\right.$ $(t), 0)$ is the number of treatment slots available at time $t$ and $r_{\max }$ is the rate of enrolling people on treatment per drug available per untreated person.

Global ART \& $\operatorname{PrEP}$. This treatment strategy is modelled with two control groups, one for ART for all infected people,

$G_{\mathrm{ART}}=\left\{I_{\mathrm{FL}}, I_{\mathrm{FM}}, I_{\mathrm{FH}}, I_{\mathrm{ML}}, I_{\mathrm{MM}}\right\}$,

and the other for PrEP for all eligible (i.e. non-low-risk) susceptible people,

$G_{\mathrm{PrEP}}=\left\{S_{\mathrm{FM}}, S_{\mathrm{FH}}, S_{\mathrm{MM}}\right\}$.

The control variables are the total number of people targeted to be on ART ( $\left.V_{\mathrm{ART}}\right)$ and PrEP $\left(V_{\mathrm{PrEP}}\right)$ at any one time. The numbers of people on ART and PrEP at time $t$ are

$$
\left.\begin{array}{l}
T_{\mathrm{ART}}(t)=\sum_{i \in\{\mathrm{F}, \mathrm{M}\} j \in\{\mathrm{L}, \mathrm{M}, \mathrm{H}\}} I_{i j \mathrm{~T}}(t), \\
T_{\operatorname{PrEP}}(t)=\sum_{i \in\{\mathrm{F}, \mathrm{M}\} j \in\{\mathrm{M}, \mathrm{H}\}} S_{i j \mathrm{~T}}(t),
\end{array}\right\}
$$

and the flows into treatment for all infected and susceptible people, respectively, are

$$
\begin{aligned}
\pi_{\mathrm{IFL}} & =\pi_{\mathrm{IFM}}=\pi_{\mathrm{IFH}}=\pi_{\mathrm{IML}}=\pi_{\mathrm{IMM}} \\
& =\pi_{\mathrm{ART}}, \pi_{\mathrm{SFM}}=\pi_{\mathrm{SFH}}=\pi_{\mathrm{SMM}}=\pi_{\mathrm{PrEP}} .
\end{aligned}
$$

The infected treated classes all receive equal levels of ART $\left(\pi_{\mathrm{ART}}\right)$; likewise, the uninfected treated classes receive equal levels of $\operatorname{PrEP}\left(\pi_{\mathrm{PrEP}}\right)$. 
The constraints on the controls are that both are positive and that their sum is less than the total amount of treatment available, $T_{\max }$ :

$$
\begin{aligned}
& V_{\mathrm{ART}} \geqslant 0, \quad V_{\mathrm{PrEP}} \geqslant 0, \\
& V_{\mathrm{ART}}+V_{\mathrm{PrEP}} \leqslant T_{\max } .
\end{aligned}
$$

Using this model for the control effort, the optimization problems we solved were to find the control variables $\left(V_{\mathrm{ART}}, V_{\mathrm{PrEP}}\right)$ that minimize one of the outcome measures - new infections [eqn (10)], total infection-years [eqn (11)], deaths due to HIV [eqn (14)], or total cost [eqn (19)] - subject to the constraints [eqn (25)]. For each proposed value of the control variables $\left(V_{\mathrm{ART}}, V_{\mathrm{PrEP}}\right)$, the value of the outcome measure was calculated by numerically solving the model differential equations [eqn (7)]. These problems, for each of the four outcome measures, were solved for varying levels of total treatment $T_{\max }$.

$A R T \&$ high-risk PrEP. We modelled this treatment strategy with three control groups: high-risk people on ART,

$G_{\mathrm{ART}, \mathrm{H}}=\left\{I_{\mathrm{FH}}\right\}$,

non-high-risk people on ART,

$G_{\mathrm{ART}, \mathrm{LM}}=\left\{I_{\mathrm{FL}}, I_{\mathrm{FM}}, I_{\mathrm{ML}}, I_{\mathrm{MM}}\right\}$,

and high-risk people on PrEP

$G_{\mathrm{PrEP}, \mathrm{H}}=\left\{S_{\mathrm{FH}}\right\}$,

with no PrEP for non-high-risk people. The control variables $\left(V_{\mathrm{ART}, \mathrm{H}}, V_{\mathrm{ART}, \mathrm{LM}}, V_{\mathrm{PrEP}, \mathrm{H}}\right)$ are the total number of people targeted to be on treatment in each of the control groups at any one time. The total numbers of people in the treatment groups at time $t$ are

$$
T_{\mathrm{ART}, \mathrm{LM}}(t)=\sum_{\substack{i \in\{\mathrm{F}, \mathrm{M}\} j \in\{\mathrm{L}, \mathrm{M}\} \\ T_{\mathrm{PrEP}, \mathrm{H}}(t)=S_{\mathrm{FHT}},}}^{T_{\mathrm{ART}, \mathrm{H}}(t),},
$$

and the flows into treatment for the control groups are

$$
\left.\begin{array}{l}
\pi_{\mathrm{IFH}}=\pi_{\mathrm{ART}, \mathrm{H}}, \\
\pi_{\mathrm{IFL}}=\pi_{\mathrm{IFM}}=\pi_{\mathrm{IML}}=\pi_{\mathrm{IMM}}=\pi_{\mathrm{ART}, \mathrm{LM}}, \\
\pi_{\mathrm{SFH}}=\pi_{\mathrm{PrEP}, \mathrm{H}}, \\
\pi_{\mathrm{SFM}}=\pi_{\mathrm{SMM}}=0 .
\end{array}\right\}
$$

The constraints on the controls are that each of them are positive and that their sum is less than the total amount of treatment available, $T_{\max }$ :

$$
\begin{aligned}
& V_{\mathrm{ART}, \mathrm{H}} \geqslant 0, \quad V_{\mathrm{ART}, \mathrm{LM}} \geqslant 0, \quad V_{\mathrm{PrEP}, \mathrm{H}} \geqslant 0, \\
& V_{\mathrm{ART}, \mathrm{H}}+V_{\mathrm{ART}, \mathrm{LM}}+V_{\mathrm{PrEP}, \mathrm{H}} \leqslant T_{\max } .
\end{aligned}
$$

Using this model for the control effort, the optimization problems we solved were to find the control variables $\left(V_{\mathrm{ART}, \mathrm{H}}, V_{\mathrm{ART}, \mathrm{LM}}, V_{\mathrm{PrEP}, \mathrm{H}}\right)$ that minimize one of the outcome measures subject to the constraints [eqn (31)]. As before, for each proposed value of the control variables, the value of the outcome measure was calculated by numerically solving the model differential equations [eqn (7)]. These problems, for each of the four outcome measures, were solved for varying levels of total treatment $T_{\max }$.

The system of ordinary differential equations [eqn (7)] was solved in Python with scipy.integrate.odeint [34], which uses LSODA (Livermore Solver for Ordinary Differential Equations) [35]. The constrained optimization problems were solved numerically in Python using scipy.optimize.fmin with method = 'COBYLA' [34], which uses the COBYLA (Constrained Optimization by Linear Approximation) algorithm [36]. To avoid finding minima which are locally but not globally optimal, the best result was kept from running the optimization routine started with several different initial guesses for the control variables. The initial guesses used were: all control variables 0 , all control variables equal to $T_{\max } / N$, one control variable equal to $T_{\max }$ and the others 0 , and 10 uniform random vectors in $\left[0, T_{\max }\right]^{N}$, where $N$ is the number of control variables.

Over a 10-year time horizon, we calculated the effectiveness of the two models (global ART \& PrEP and high-risk ART \& PrEP) according the four different outcome measures: new infections, total infection-years, total deaths due to AIDS and total cost. New infections is the total incidence over the 10-year span. Total infection-years is the number of infected people, both treated and untreated, at each time, summed over the time period. Deaths due to AIDS is simply the total number of deaths over the time period. The total cost sums costs of infections, deaths and treatment.

To evaluate the sensitivity of our results to parameter uncertainty, we computed the infection-years, costs, new infections deaths and cost arising from a 50\% increased and $50 \%$ decrease in the default parameters of: mediumrisk contact rate $\left(\beta_{\mathrm{M}}\right)$; high-risk contact rate $\left(\beta_{\mathrm{H}}\right)$; rate of enrolling people on treatment $\left(r_{\max }\right)$; and efficacy of PrEP at preventing transmission $\left(\theta_{\mathrm{PrEP}}\right)$. Even though a 

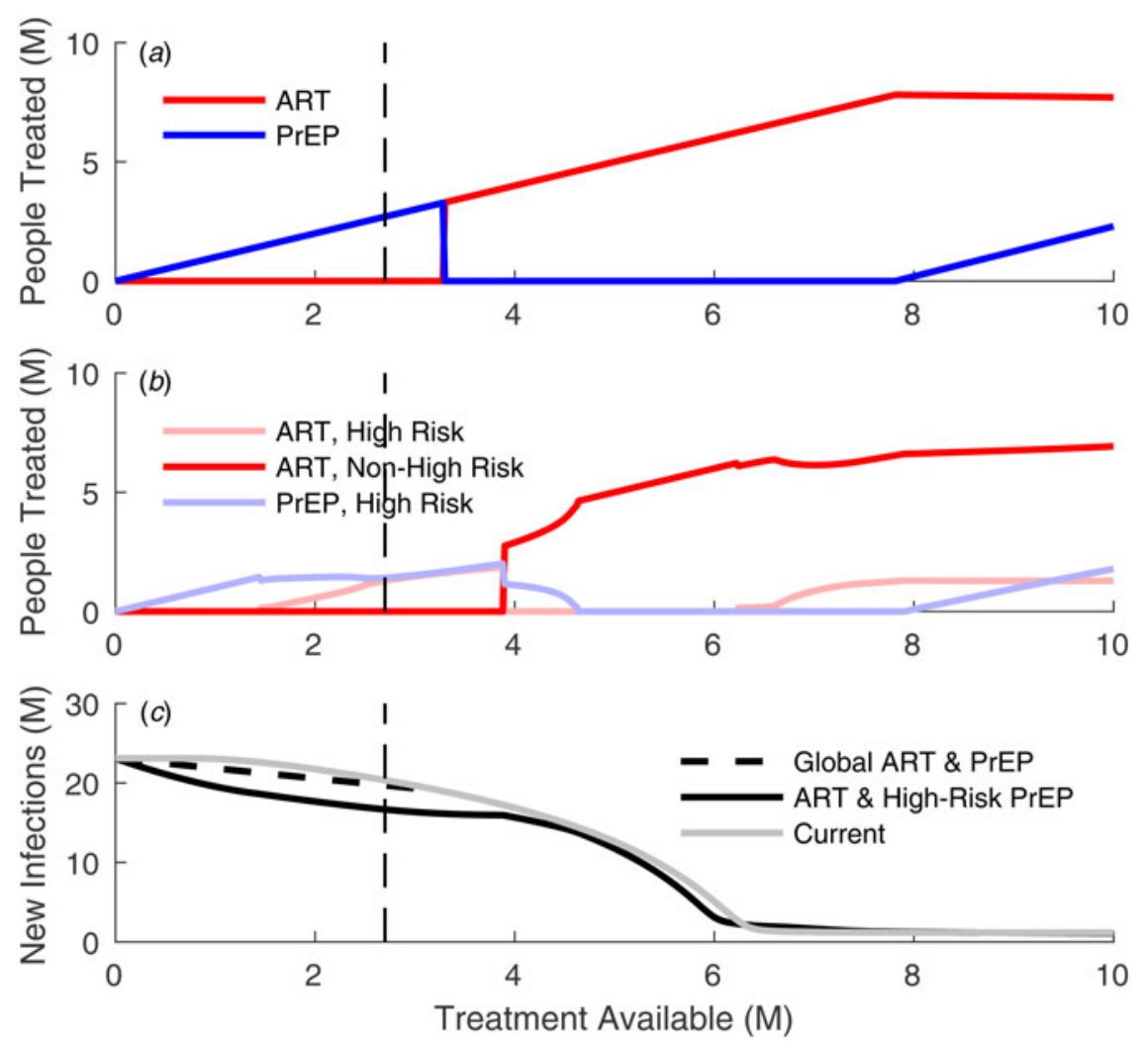

Fig. 2. Treatment allocations that minimize new infections and their impact. (a) Under the global ART \& PrEP strategy, people are treated independently of their risk group, only depending on their HIV status. We found the allocation of ART to HIV-positive people (red line) and PrEP to HIV-negative people (blue line) minimizes new infections over the 10-year model period, as the amount of treatment available varies. (b) The ART \& high-risk PrEP strategy allocates ART at different levels depending on whether people have high risk of transmission or not, and allocates PrEP to high-risk people. We found the allocation of ART to high-risk HIV-positive people (pink line), ART to non-high-risk HIV-positive people (red line), and PrEP to high-risk HIV-negative people (light blue line) minimizes new infections as the amount of treatment available varies. (c) We compared the minimized number of new infections over the 10-year model period vs. treatment available for the global ART \& PrEP strategy (black dashed line) and the ART \& high-risk PrEP strategy (black solid line) to South Africa's current policy (grey line) of allocating ART to HIV-positive people without regard for transmission risk and allocating no PrEP to HIV-negative people. The vertical dashed line is the current level of drug availability in South Africa.

$50 \%$ increase in $\theta_{\mathrm{PrEP}}$ is $>100 \%$, we used $100 \%$ since that is the maximum efficiency.

\section{RESULTS}

We simulated our model to determine the levels of treatment that minimize each of the outcome measures and to determine the effect of applying the optimal treatment strategy over a 10 -year period. We examined the optimal allocation of ART and PrEP to people independent of their risk status (global ART \& PrEP). In order to evaluate the current CDC treatment recommendations, we also evaluated the optimal allocation of ART and PrEP to high-risk people in addition to ART for the remainder of the population (ART \& high-risk PrEP). We also compared the effectiveness of global ART \& PrEP and ART \& high-risk PrEP with the current treatment strategy in South Africa of only giving ART and the UNAIDS's 90-90-90 goals, in terms of reducing new infections, infection-years, deaths due to HIV, and cost.

With the global ART \& PrEP control strategy, the optimal treatment allocations that minimized each of the four outcome measures showed similar patterns below about 7.5 million (M) available treatment spots (Figs $2 a-5 a$ ), i.e. only PrEP was used at lowtreatment availability and only ART was used at higher levels of treatment availability, with the threshold level of treatment for switching ranging from 

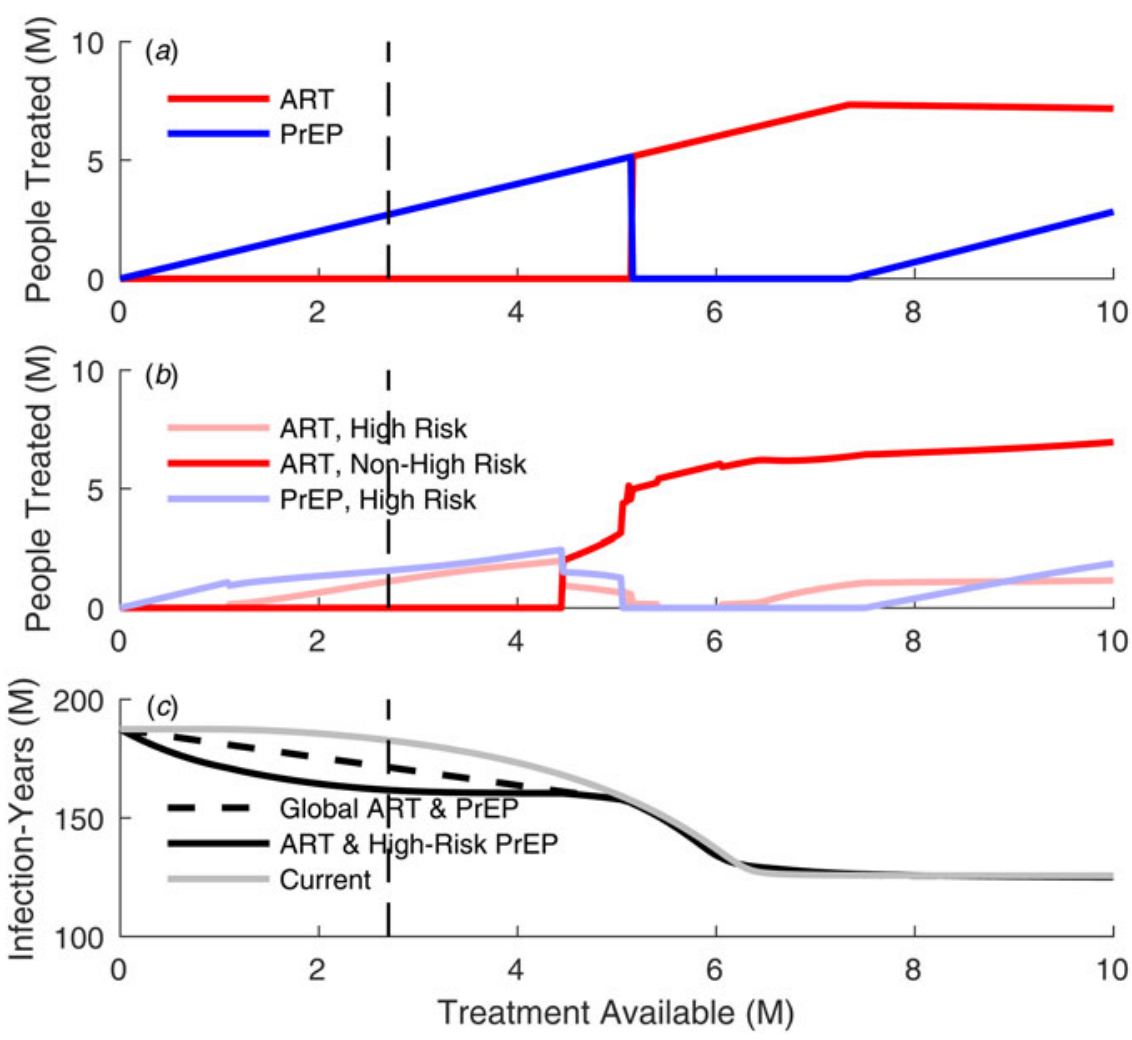

Fig. 3. Treatment allocations that minimize infection-years and their impact. Infection-years is the number of person-years lived by HIV-infected people over the 10-year model period. (a) Under the global ART \& PrEP strategy, people are treated independently of their risk group, only depending on their HIV status. We found the allocation of ART to HIV-positive people (red line) and PrEP to HIV-negative people (blue line) minimizes infection-years over the 10-year model period as the amount of treatment available varies. (b) The ART \& high-risk PrEP strategy allocates ART at different levels depending on whether people have high risk of transmission or not, and allocates PrEP to high-risk people. We found the allocation of ART to high-risk HIV-positive people (pink line), ART to non-high-risk HIV-positive people (red line), and PrEP to high-risk HIV-negative people (light blue line) minimizes infection-years as the amount of treatment available varies. $(c)$ We compared the minimized number of infection-years over the 10-year model period vs. treatment available for the global ART \& PrEP strategy (black dashed line) and the ART \& high-risk PrEP strategy (black solid line) to South Africa's current policy (grey line) of allocating ART to HIV-positive people without regard for transmission risk and allocating no PrEP to HIV-negative people. The vertical dashed line is the current level of drug availability in South Africa.

about $2 \cdot 0 \mathrm{M}$ to $5 \cdot 3 \mathrm{M}$, depending on the outcome measure. Above $7 \cdot 3-7 \cdot 8 \mathrm{M}$ available treatment spots, two patterns emerged, adding PrEP in the cases of minimizing new infections and infection-years, and continuing to add ART, not PrEP, when minimizing deaths and cost.

For the ART \& high-risk PrEP control strategy, minimizing the four outcome measures again showed similar patterns (Figs $2 b-5 b$ ). At low treatment availability, treatment was prioritized to high-risk people, first starting with PrEP and then adding on, or switching to in the case of minimizing deaths, ART. As available treatment increased, the treatment strategy switched to predominantly or entirely ART for non-high-risk people, with the thresholds occurring between $2 \cdot 8 \mathrm{M}$ and $5 \cdot 8 \mathrm{M}$ available treatment spots, depending on the outcome measure. At even higher levels of treatment, above $6 \cdot 0-6 \cdot 4 \mathrm{M}$, ART was added for high-risk people for all outcome measures. Finally, above $7 \cdot 5-7 \cdot 9 \mathrm{M}$ available treatment spots, high-risk PrEP was added when minimizing new infections and infection-years.

Over the 10-year model period, increasing the amount of available treatment led to reductions in all four outcome measures (Figs $2 c-5 c$ ), as expected. Substantial decreases in all outcome measures were seen for increasing treatment until around $6 \mathrm{M}$ available treatment spots. Increased treatment beyond this level resulted in very small gains to all outcome measures. For example, the number of deaths over 

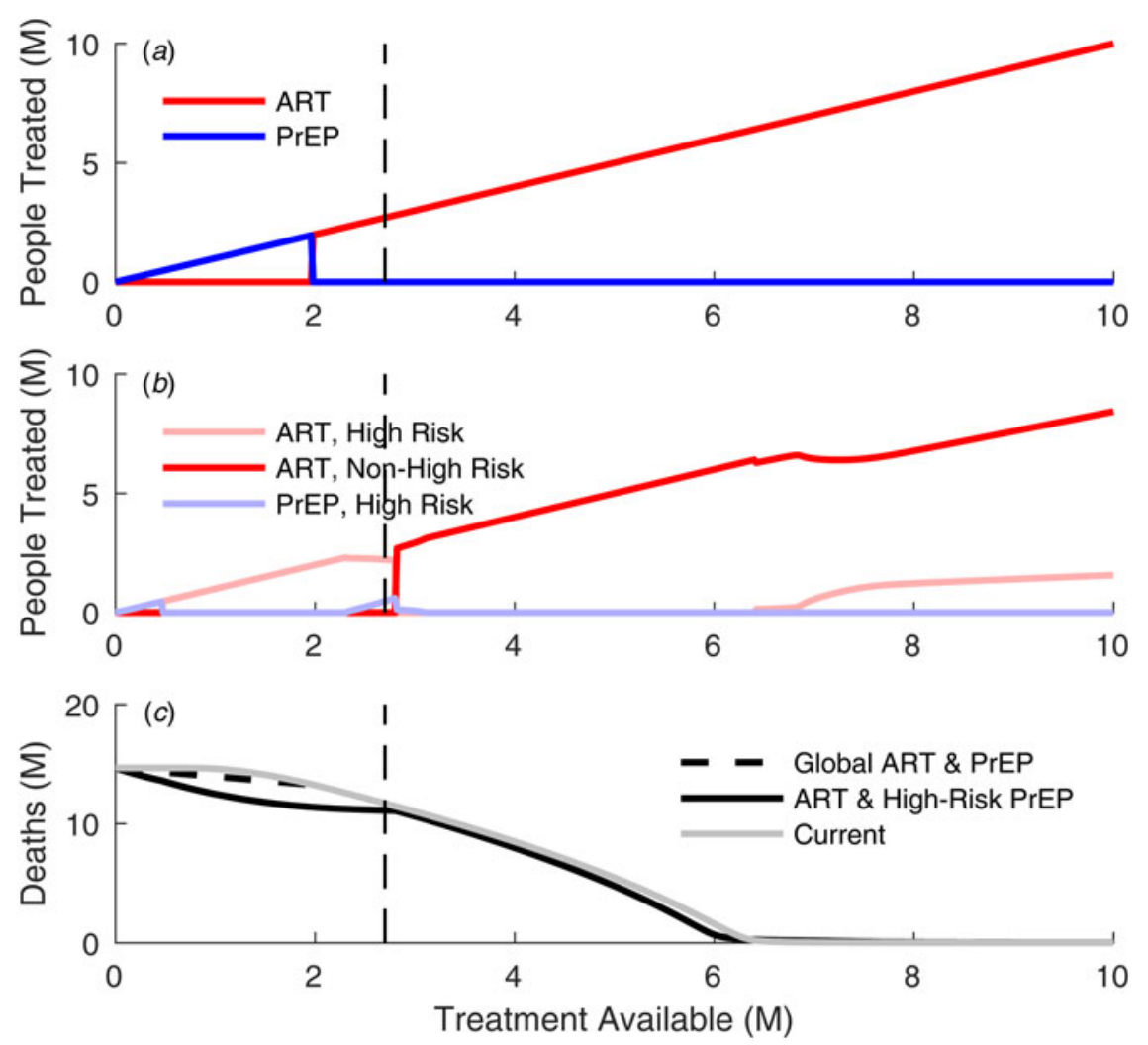

Fig. 4. Treatment allocations that minimize HIV-related deaths and their impact. (a) Under the global ART \& PrEP strategy, people are treated independently of their risk group, only depending on their HIV status. We found the allocation of ART to HIV-positive people (red line) and PrEP to HIV-negative people (blue line) minimizes deaths over the 10-year model period as the amount of treatment available varies. (b) The ART \& high-risk PrEP strategy allocates ART at different levels depending on whether people have high risk of transmission or not, and allocates PrEP to high-risk people. We found the allocation of ART to high-risk HIV-positive people (pink line), ART to non-high-risk HIV-positive people (red line), and PrEP to high-risk HIV-negative people (light blue line) minimizes deaths as the amount of treatment available varies. $(c)$ We compared the minimized number of deaths over the 10-year model period vs. treatment available for the global ART \& PrEP strategy (black dashed line) and the ART \& high-risk PrEP strategy (black solid line) to South Africa's current policy (grey line) of allocating ART to HIV-positive people without regard for transmission risk and allocating no PrEP to HIV-negative people. The vertical dashed line is the current level of drug availability in South Africa.

the 10 years fell from $14 \cdot 7 \mathrm{M}$ with no treatment to about 3000 with $6.5 \mathrm{M}$ available treatment spots and increased treatment above $6.5 \mathrm{M}$ did not change the number of deaths by much. For low drug availability (i.e. $2 \cdot 5-5 \cdot 3 \mathrm{M}$ ), the ART \& high-risk PrEP control strategy decreased all outcome measures faster than either global ART \& PrEP or the current strategy of global ART and, for minimizing new infections and minimizing deaths, the global ART \& PrEP strategy preformed only slightly better than the current strategy. For higher levels of treatment, all three strategies performed similarly for all outcome measures.

In South Africa currently, $42 \%$ or $2.7 \mathrm{M}$ of the $6.5 \mathrm{M}$ HIV-infected people are on ART. To implement the UNAIDS's 90-90-90 goal, 5·8M people would need to be on ART currently. With the global ART \&
PrEP control strategy, allocating all of the available treatment as ART and none to PrEP minimized all four outcome measures (Table 4). These optimal allocations, all ART and no PrEP, are exactly the current strategy in South Africa. With the ART \& high-risk PrEP strategy, allocating all of the available treatment to non-high-risk ART minimized new infections, infection-years, and deaths, while cost was minimized by allocating 150000 treatment spots to high-risk ART and the remainder to non-high-risk ART. The ART \& high-risk PrEP strategy performed better than global ART \& PrEP for all four outcome measures, but only slightly so for infection-years and cost.

For both treatment strategies, $r_{\max }$ is the least sensitive parameter while $\beta_{\mathrm{M}}$ is the most sensitive parameter. A $50 \%$ increase/decrease in the default value of 

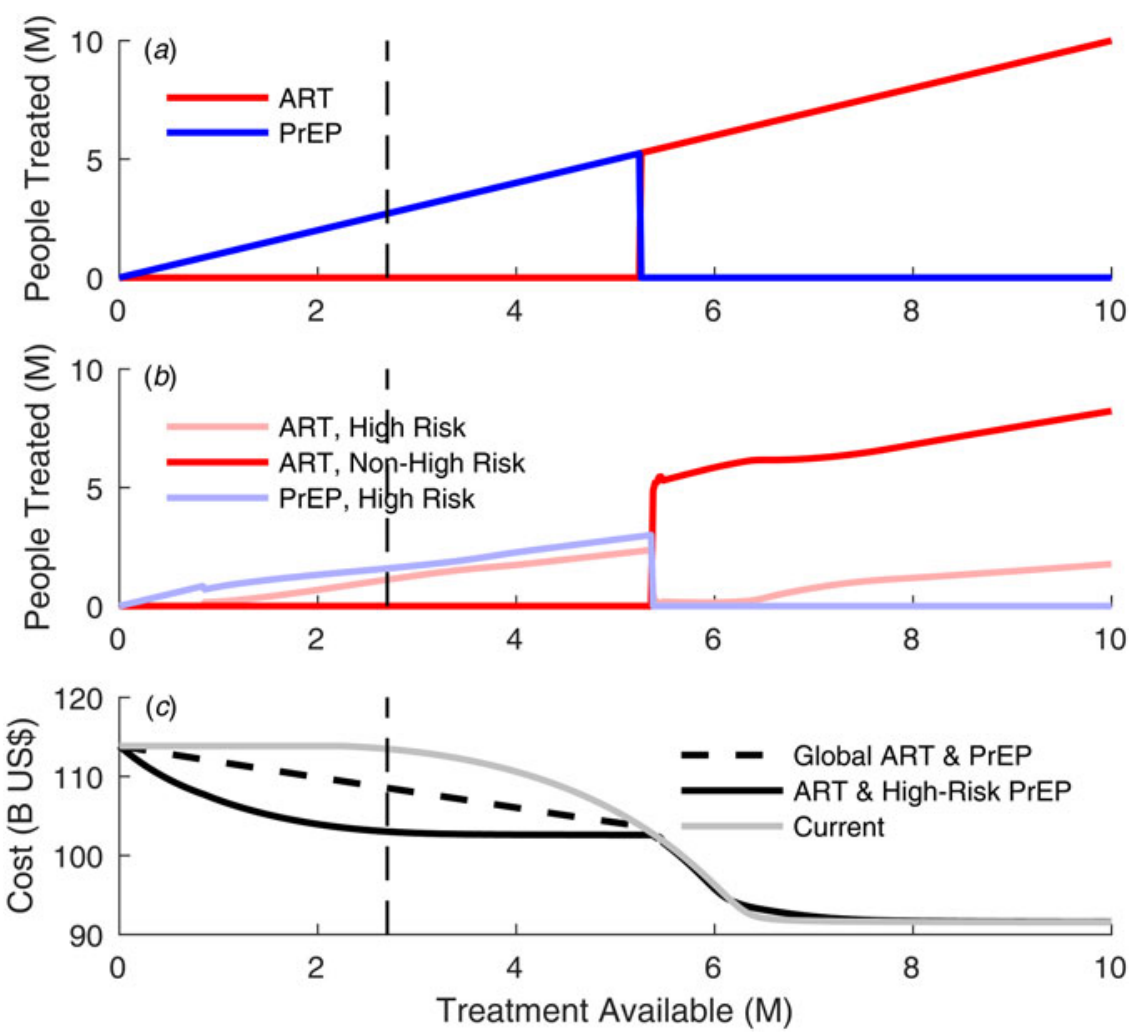

Fig. 5. Treatment allocations that minimize financial cost. Financial cost is the total financial cost to society of HIV infections, HIV-related deaths, and providing ART and PrEP. (a) Under the global ART \& PrEP strategy, people are treated independently of their risk group, only depending on their HIV status. We found the allocation of ART to HIV-positive people (red line) and PrEP to HIV-negative people (blue line) minimizes cost over the 10-year model period as the amount of treatment available varies. (b) The ART \& high-risk PrEP strategy allocates ART at different levels depending on whether people have high risk of transmission or not, and allocates PrEP to high-risk people. We found the allocation of ART to high-risk HIV-positive people (pink line), ART to non-high-risk HIV-positive people (red line), and PrEP to high-risk HIV-negative people (light blue line) minimizes cost as the amount of treatment available varies. (c) We compared the minimized number of cost over the 10-year model period vs. treatment available for the global ART \& PrEP strategy (black dashed line) and the ART \& high-risk PrEP strategy (black solid line) to South Africa's current policy (grey line) of allocating ART to HIV-positive people without regard for transmission risk and allocating no PrEP to HIV-negative people. The vertical dashed line is the current level of drug availability in South Africa.

Table 4. Optimal treatment allocations at 5.8M available treatment spots (i.e. enough treatment for $90 \%$ coverage of infected people) and their effectiveness for the four outcome measures under both the global ART \& PrEP and ART \& high-risk PrEP control strategies

\begin{tabular}{|c|c|c|c|c|c|c|c|}
\hline \multirow[b]{2}{*}{$\begin{array}{l}\text { Objective } \\
\text { minimized }\end{array}$} & \multicolumn{3}{|c|}{ Global ART \& PrEP } & \multicolumn{3}{|c|}{ ART \& high-risk PrEP } & \multirow[b]{2}{*}{$\begin{array}{l}\text { Objective } \\
\text { value }\end{array}$} \\
\hline & $\begin{array}{l}\text { ART } \\
(\mathrm{M})\end{array}$ & $\begin{array}{l}\text { PrEP } \\
(\mathrm{M})\end{array}$ & $\begin{array}{l}\text { Objective } \\
\text { value }\end{array}$ & $\begin{array}{l}\text { High-risk } \\
\text { ART (M) }\end{array}$ & $\begin{array}{l}\text { High-risk } \\
\text { PrEP (M) }\end{array}$ & $\begin{array}{l}\text { Non-high-risk } \\
\text { ART (M) }\end{array}$ & \\
\hline New infections & $5 \cdot 8$ & 0 & $6 \cdot 9 \mathrm{M}$ & 0 & 0 & $5 \cdot 8$ & $5 \cdot 0 \mathrm{M}$ \\
\hline Infection-years & $5 \cdot 8$ & 0 & $142 \mathrm{M}$ & 0 & 0 & $5 \cdot 8$ & $139 \mathrm{M}$ \\
\hline Deaths & $5 \cdot 8$ & 0 & $2 \cdot 4 \mathrm{M}$ & 0 & 0 & $5 \cdot 8$ & $1 \cdot 4 \mathrm{M}$ \\
\hline Cost & $5 \cdot 8$ & 0 & $\$ 98.4 \mathrm{~B}$ & $0 \cdot 1$ & 0 & $5 \cdot 7$ & $\$ 98.0 \mathrm{~B}$ \\
\hline
\end{tabular}

$r_{\max }$ leads to almost no changes in the value of the all four outcomes measures. In the case of $\beta_{\mathrm{M}}$ the increase/decrease corresponds to an increase/decrease of $16-49 \%$ in the outcomes measures. Increasing $\beta_{\mathrm{M}}$ and $\beta_{\mathrm{H}}$ leads to corresponding increases in the respective outcome measures and decreasing them also leads 

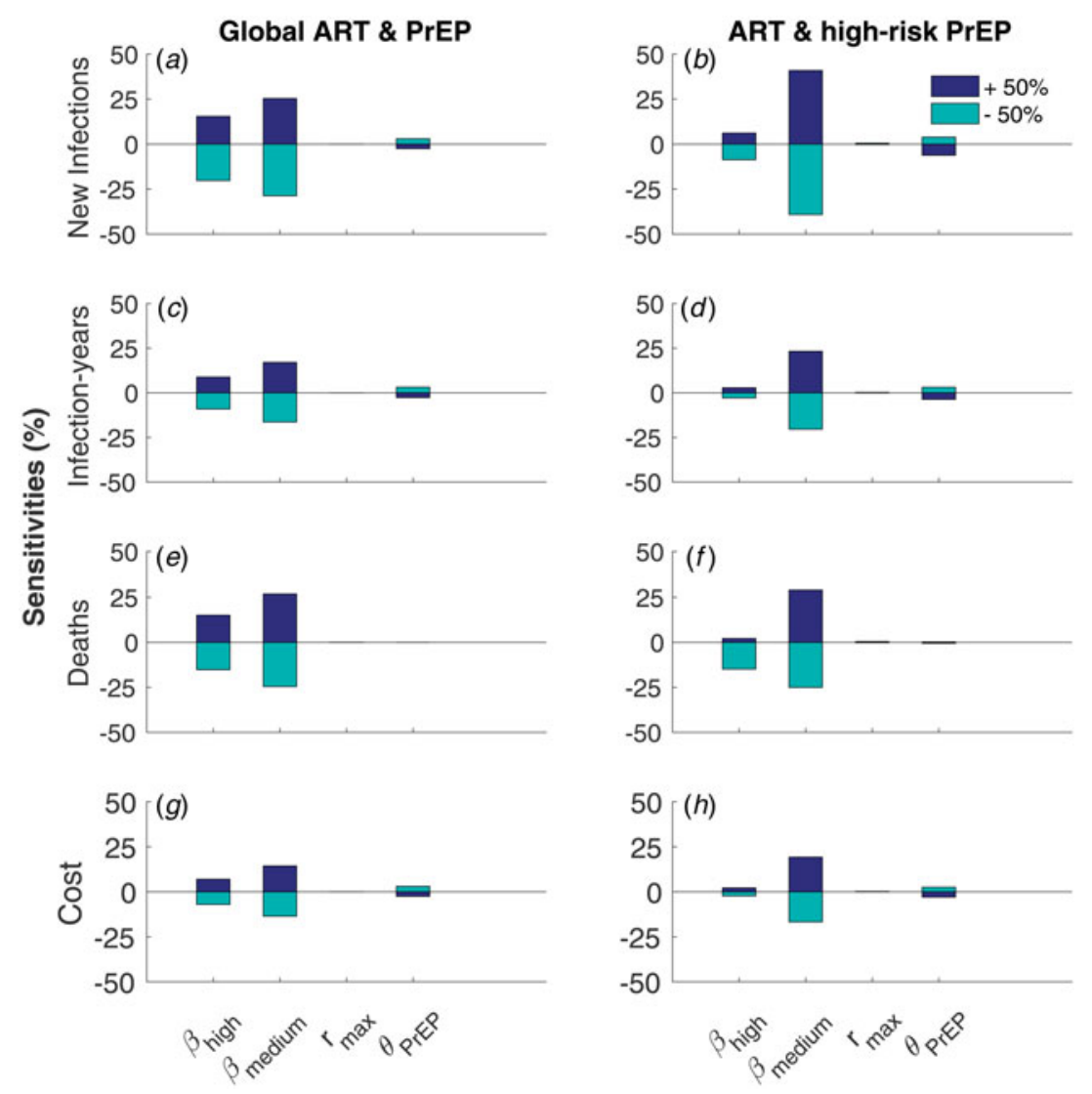

Fig. 6. Sensitivity of different outcome measures to model parameters for both allocation strategies. The parameters were increased by $50 \%$ (dark blue bars) and decreased by $50 \%$ (cyan bars) from their default values, the optimal allocation we calculated for each outcome measure (rows) and both allocation strategies (columns), and the relative change in each outcome measure was recorded. The left panels $(a, c, e, g)$ represent the global ART \& PrEP strategy, while the right panels $(b, d, f, h)$ represent the ART \& high-risk PrEP strategy.

to corresponding decreases in the respective outcome measures. Unsurprisingly, the opposite is observed for $\theta_{\mathrm{PrEP}}$ where an increase in $\theta_{\mathrm{PrEP}}$ leads to a decrease in the respective outcome measures and a decrease leads to an increase in the respective outcome measures (Fig. 6).

\section{DISCUSSION}

Using a mathematical model of heterosexual HIV transmission, we determined two treatment strategies that minimize new infections, infection-years, AIDSrelated deaths and cost. We found that when PrEP and ART are given to the general population at different levels (our global PrEP \& ART strategy), only PrEP should be used at low treatment availability, but only ART should be used at higher levels, with the thresholds for switching from PrEP to ART differing for the four outcome measures. At very high levels of drug availability, adding PrEP minimizes new infections and infection-years, while continuing to use only ART minimizes deaths and cost. When PrEP and ART are given to high-risk people and ART to non-high-risk people (our ART \& high-risk PrEP strategy), we found that at low-treatment availability only high-risk people should be treated; non-high-risk people should only be treated above threshold levels of treatment that vary by outcome measure. At high-treatment availability, treating both high-risk and non-high risk individuals becomes the optimal allocation for all outcome measures.

The reason why PrEP is preferred at drug availability might be because giving all the limited treatments as ART at low drug availability will result in the protection from infection of only the immediate uninfected partners of the people treated. Due to homogeneous mixing these uninfected partners can still become infected by other untreated people in the population. Therefore giving all these drugs as PrEP is better policy. The efficacy of PrEP is higher 
than the efficacy of ART in preventing infections; this could also account for the preference of PrEP over ART at low drug availability.

We also evaluated the UNAIDS recent 90-90-90 target and found that the current ART-only treatment strategy of South Africa and indeed most resourcelimited countries is optimal or near optimal at $90 \%$ treatment coverage for minimizing all four outcome measures. The one exception was that allocating 150000 treatment spots for ART to high-risk people and the remainder to ART for non-high-risk people minimized cost.

Resource-limited countries are likely to have low treatment availability: for example with South Africa's current level of treatment availability $(2 \cdot 7 \mathrm{M}$ slots), PrEP to the general population (for global ART \& PrEP) or treatment of only sex workers with ART \& PrEP (for ART \& high-risk PrEP) is most effective at reducing new infections, infection-years, and cost, while using the treatment as ART to either the general population (global ART \& PrEP) or ART to non-high-risk people (ART \& high-risk PrEP) best reduces deaths.

Our model allows for the possibility of ceasing to treat people who have been on treatment for some time, reallocating their drugs to others for the benefit of the whole population. This is an ethically complicated situation for many reasons: e.g., doctors' oaths to do no harm, and the fact that the people whose treatment is ending have been reliably following the treatment regimen, while the people to be added may not do so.

Allocations that prioritize treatment of commercial sex workers (CSWs) may be difficult to implement. CSWs are often stigmatized and their actions are frequently illegal, which may present challenges to getting them out of the shadows for treatment. Even when CSWs do come forward for treatment, prioritizing treatment to them will mean withholding treatment from non-high-risk people, which may be a difficult decision for policymakers to justify. However, the targeting of preventive treatment to high-risk people has found to be a effective strategy to control sexually transmitted diseases [37-39]. Moreover, donors concerned with women's health may also expect access to PrEP for women at risk. Most importantly, we believe that the huge savings in HIV mortality, morbidity, and cost clearly justify such policies.

We hope that this work provides some guidance for policymakers in determining how to allocate HIV treatment to maximize their benefit.

\section{SUPPLEMENTARY MATERIAL}

For supplementary material accompanying this paper visit https://doi.org/10.1017/S0950268816002958.

\section{ACKNOWLEDGEMENTS}

G.A. and J.M. were supported by US National Institute of General Medical Sciences award 5U01GM070694-11 and by funds from Oregon State University. A.P. was supported by the Brown Coxe Postdoctoral Fellowship and a grant from the Notsew Orm Sands Foundation.

\section{DECLARATION OF INTEREST}

None.

\section{REFERENCES}

1. UNAIDSorg. AIDSinfo j UNAIDS (http://www.un AIDS.org/en/dataanalysis/datatools/AIDSinfo). Accessed 25 September 2015.

2. Alary M, Lowndes CM. The central role of clients of female sex workers in the dynamics of heterosexual HIV transmission in sub-Saharan Africa. AIDS 2004; 18: 945-947.

3. Shelton JD. Ten myths and one truth about generalised HIV epidemics. Lancet, 2007; 370: 1809-1811.

4. Halperin DT, Epstein H. Concurrent sexual partnerships help to explain Africa's high HIV prevalence: implications for prevention. Lancet 2004; 364: 4-6.

5. Bráu N, et al. Slower fibrosis progression in HIV/ HCV-coinfected patients with successful HIV suppression using antiretroviral therapy. Journal of Hepatology 2006; 44: 47-55.

6. van Sighem AI, et al. Mortality and progression to AIDS after starting highly active antiretroviral therapy. AIDS 2003; 17: 2227-2236.

7. Porco TC, et al. Decline in HIV infectivity following the introduction of highly active antiretroviral therapy. AIDS 2004; 18: 81-88.

8. Quinn TC, et al. Viral load and heterosexual transmission of human immunodeficiency virus type 1 . New England Journal of Medicine 2000; 342: 921-929.

9. Gray RH, et al. Probability of HIV-1 transmission per coital act in monogamous, heterosexual, HIV-1-discordant couples in Rakai, Uganda. Lancet 2001; 357: 11491153.

10. Cohen J. HIV treatment as prevention. Science 2011; 334: 1628-1628.

11. Grant RM, et al. Preexposure chemoprophylaxis for HIV prevention in men who have sex with men. New England Journal of Medicine 2010; 363: 2587-2599.

12. Baeten JM, et al. Antiretroviral prophylaxis for HIV prevention in heterosexual men and women. New England Journal of Medicine 2012; 367: 399-410.

13. Abdool Karim Q, et al. Effectiveness and safety of tenofovir gel, an antiretroviral microbicide, for the 
prevention of HIV infection in women. Science 2010; 329: $1168-1174$.

14. Centers for Disease Control and Prevention. Preexposure prophylaxis for the prevention of HIV infection in the United States-2014 (http://www.cdc.gov/HIV/pdf/ guidelines/PrEPguidelines2014.pdf). Accessed 25 September 2015.

15. Montaner JS, et al. The case for expanding access to highly active antiretroviral therapy to curb the growth of the HIV epidemic. Lancet 2006; 368: 531-536.

16. Crowley S. Preventing HIV transmission with antiretrovirals. Bulletin of the World Health Organization 2009; 87: 488-488.

17. De Cock KM, et al. Can antiretroviral therapy eliminate HIV transmission? Lancet, 2009; 373: 7-9.

18. Cohen J. HIV treatment as prevention. Science 2011; 334: $1628-1628$.

19. World Health Organization. Consolidated guidelines on the use of antiretroviral drugs for treating and preventing HIV infection (http://www.who.int/hiv/pub/guidelines/arv2013/en/). Accessed 25 September 2015.

20. UNAIDSorg 90-90-90: an ambitious treatment target to help end the AIDS epidemic (http://www.unAIDS. org/en/resources/documents/2014/90-90-90). Accessed 25 September 2015.

21. Richter ML, et al. Characteristics, sexual behaviour and risk factors of female, male and transgender sex workers in South Africa. South African Medical Journal 2013; 103.

22. De Gruttola V, et al. Modeling the relationship between survival and CD4 lymphocytes in patients with AIDS and AIDS-related complex. JAIDS Journal of Acquired Immune Deficiency Syndromes 1993; 6: 359-365.

23. De Gruttola V, Tu XM. Modelling progression of CD4-lymphocyte count and its relationship to survival time. Biometrics 1994; 50: 1003-1014.

24. Simbayi L, et al. South African National HIV Prevalence, Incidence and Behaviour Survey, 2012. Cape Town, South Africa: HSRC Press, 2014.

25. Statistics South Africa. Mid-year population estimates 2014 (http://www.statssa.gov.za/publications/P0302/ P03022014.pdf). Accessed 21 October 2015.

26. Wiley JA, Herschkorn SJ, Padian NS. Heterogeneity in the probability of HIV transmission per sexual contact: the case of male-to-female transmission in penile-vaginal intercourse. Statistics in Medicine 1989; 8: 93-102.

27. Cohen MS, et al. Prevention of HIV-1 infection with early antiretroviral therapy. New England Journal of Medicine 2011; 365: 493-505.

28. Cleary SM, McIntyre D, Boulle AM. The costeffectiveness of antiretroviral treatment in Khayelitsha, South Africa - a primary data analysis. Cost Effectiveness and Resource Allocation 2006; 4: 20.

29. Médecins Sans Frontières. Untangling the web of antiretroviral price reductions: 14th edn - July 2011 (http:// d2pd3b5abq75bb.cloudfront.net/2012/07/16/14/42/23/52/ UTW_14_ENG_July2011.pdf). Accessed 21 Oct 2015.

30. Gold M, et al. Cost-Effectiveness in Health and Medicine. New York: Oxford University Press, 1996.

31. Chen RY, et al. Distribution of health care expenditures for HIV-infected patients. Clinical Infectious Diseases 2006; 42: 1003-1010.

32. Dixit AK, Pindyck RS. Investment under Uncertainty. Princeton, NJ: Princeton University Press, 1994.

33. Centers for Disease Control and Prevention. Preexposure prophylaxis for the prevention of HIV infection in the United States-2014: clinical providers' supplement (http://www.cdc.gov/hiv/pdf/prepprovidersupplement2014.pdf). Accessed 25 September 2015.

34. Jones E, et al. SciPy: open source scientific tools for Python (http://www.scipy.org). Accessed 25 September 2015.

35. Hindmarsh AC. Brief description of ODEPACK - a systematized collection of ODE solvers double precision version (http://www.netlib.org/odepack/opkd-sum). Accessed 25 September 2015.

36. Powell M. Direct search algorithms for optimization calculations. Acta Numerica 1998; 7: 287-336.

37. Hethcote HW, Yorke JA. Gonorrhea Transmission Dynamics and Control. Springer-Verlag, 1984.

38. Lowndes CM, et al. Management of sexually transmitted diseases and HIV prevention in men at high risk: targeting clients and non-paying sexual partners of female sex workers in Benin. AIDS 2000; 14: 2523-2534.

39. Alary M, et al. Decline in the prevalence of HIV and sexually transmitted diseases among female sex workers in Cotonou, Benin, 1993-1999. AIDS 2002; 16: 463470 . 